\title{
Very low concentration of lipopolysaccharide can induce the production of various cytokines and chemokines in human primary monocytes
}

\author{
Ratthakorn Chaiwut ${ }^{1}$ and Watchara Kasinrerk ${ }^{1,2^{*}}$ (D)
}

\begin{abstract}
Objective: Lipopolysaccharide (LPS), a component of gram-negative bacteria, is a potent innate immune stimulus. The interaction of LPS with innate immune cells induces the production of proinflammatory cytokines and chemokines, thereby leading to the control of infection. In the present study, we investigated the effect of a wide range of LPS concentrations on the regulation of various proinflammatory cytokines and chemokines in human primary monocytes and T lymphocytes.

Results: We demonstrated that a very low concentration of LPS could regulate the production of cytokines and chemokines in monocytes but not T lymphocytes. Unexpectedly, very low concentrations of LPS (0.0025 and $0.005 \mathrm{ng} / \mathrm{mL}$ ) could induce TNF-a and IL-6 production, respectively, in monocytes. Our findings provide evidence that in the presence of monocytes, even very low endotoxin contamination could induce cytokine production. We suggest that the recombinant proteins used to investigate immune functions must be thoroughly screened for endotoxins using a highly sensitive method.
\end{abstract}

Keywords: Chemokine, Innate immunity, Lipopolysaccharide, Monocyte, Proinflammatory cytokine

\section{Introduction}

Lipopolysaccharide (LPS) is a component of the outer membrane of gram-negative bacteria. The interaction of LPS with innate immune cells, especially monocytes and macrophages, triggers inflammatory responses to control pathogen infection [1-3]. The activation of innate immune cells by LPS is initiated by the sequential actions of LPS-binding protein (LBP), CD14, MD-2, and TLR-4 [1, 4-7]. LBP, in concert with CD14, presents LPS molecules to the MD-2/TLR4 receptor complex, which leads to the activation of MyD88- and TRIF-dependent signalling pathways $[1,6,8]$. The MyD88-dependent signalling

\footnotetext{
*Correspondence: watchara.k@cmu.ac.th

${ }^{1}$ Division of Clinical Immunology, Department of Medical Technology, Faculty of Associated Medical Sciences, Chiang Mai University, Chiang Mai, Thailand

Full list of author information is available at the end of the article
}

pathway facilitates the rapid activation of NF-kB, thereby leading to the production of various proinflammatory cytokines and chemokines. Conversely, TRIF-dependent pathways lead to the activation of the transcription factor IRF3 and promote the production of type I IFN and IFNdependent chemokines. Therefore, activation of innate immune cells by LPS initiates cross-talk between innate and adaptive immune responses $[1,9,10]$.

Currently, many recombinant proteins are used to investigate immune functions. Many of these proteins, which are generated in-house and are commercially available, are produced in Escherichia coli and may be contaminated by endotoxin. The amount of endotoxin contamination is usually stated by researchers or on the data sheets accompanying commercial products, and that the endotoxin level is less than 1 endotoxin unit (EU), which is equivalent to $0.1 \mathrm{ng}$ of E. coli LPS per $\mu \mathrm{g}$ of protein [11]. However, concerns about monocytes, which 
are highly sensitive to LPS, have been raised $[1,2,12]$. Residual endotoxin contamination in the recombinant protein used might be sufficient to activate monocytes [1, 12 ] and lead to misinterpretation when using the recombinant protein to validate immune responses. In the present study, we assessed the effect of a wide range of LPS concentrations on the production of various proinflammatory cytokines and chemokines in human primary monocytes and T lymphocytes. The range of LPS concentrations and the cytokines/chemokines studied were different from those documented. We demonstrated that a very low concentration of LPS $(0.0025 \mathrm{ng} / \mathrm{mL})$ was able to activate monocytes. These results suggest the importance of awareness of LPS contamination in any substance when working with monocytes.

\section{Main text \\ Methods \\ Antibodies, reagents, and cells}

LPS from Escherichia coli serotype 055:B5 was purchased from Sigma-Aldrich (Taufkirchen, Germany). The anti-CD3 monoclonal antibody $(\mathrm{mAb})$ clone OKT3 and anti-CD28 mAb clone L293 were purchased from Ortho Pharmaceuticals (Raritan, NJ, USA) and BD Bioscience (San Jose, CA, USA), respectively. FITC-labelled antiCD3 mAbs were purchased from BD Biosciences (San Jose, CA, USA). PerCP-labelled anti-CD14 mAbs and PE-conjugated anti-IFN- $\gamma$, anti-TNF- $\alpha$, anti-IL- 6 , and anti-IL-10 were purchased from BioLegend (San Diego, CA, USA). PE-conjugated anti-GM-CSF, anti-IL-1 $\beta$, antiCCL2, anti-CCL3, anti-CCL4, and anti-CXCL10 mAbs were purchased from Miltenyi Biotech (Bergisch Gladbach, Germany). Brefeldin A and monensin were purchased from BioLegend (San Diego, CA, USA).

Peripheral blood mononuclear cells (PBMCs) were isolated from healthy donors using Ficoll-Hypaque (IsoPrep; Robbins Scientific Corporation, Sunnyvale, CA, USA) gradient centrifugation.

\section{LPS stimulation}

PBMCs were seeded in a 24-well culture plate (Costar; Corning, NY, USA) at a concentration of $1 \times 10^{6}$ cells per well. The cells were stimulated with various concentrations of LPS, immobilized anti-CD3 mAbs (25 ng/ $\mathrm{mL})$ and soluble anti-CD28 mAbs $(50 \mathrm{ng} / \mathrm{mL})$ as a T cell activation control or RPMI containing $10 \% \mathrm{FBS}$ as an unstimulated control. Cells were incubated at $37^{\circ} \mathrm{C}$ in a humidified incubator with $5 \% \mathrm{CO}_{2}$. After $1 \mathrm{~h}$ of incubation, $1 \mu \mathrm{g} / \mathrm{mL}$ brefeldin $\mathrm{A}$ and $1 \mu \mathrm{M}$ monensin were added and continuously incubated for $5 \mathrm{~h}$. Cells were then harvested for further intracellular staining.

\section{Intracellular cytokine staining}

After being stimulated, the cells were washed twice with phosphate-buffered saline (PBS) containing 1\% BSA and $0.1 \% \mathrm{NaN}_{3}$. The cells were then fixed with $4 \%$ paraformaldehyde for $15 \mathrm{~min}$ at room temperature, washed and blocked with $10 \%$ heat-inactivated human blood group $A B$ serum. The blocked cells were then stained with fluorochrome-conjugated anti-CD3, anti-CD14, anti-IFN- $\gamma$, anti-TNF- $\alpha$, anti-GM-CSF, anti-IL- $1 \beta$, antiIL-6, anti-IL-10, anti-CCL2, anti-CCL3, anti-CCL4, and anti-CXCL10 mAbs or isotype-matched control mAbs for $30 \mathrm{~min}$ at $4{ }^{\circ} \mathrm{C}$. The cells were then washed and fixed with PBS containing $1 \%$ paraformaldehyde. The stained cells were quantified using a flow cytometer (BD Accuri ${ }^{\mathrm{TM}}$ C6 flow cytometer; BD Biosciences) and analysed using FlowJo software. During analysis, monocytes and T lymphocytes were gated based on the expression of CD14 and $\mathrm{CD} 3$, respectively.

\section{Statistical analysis}

The data are expressed as the mean \pm standard error of the mean (SEM). All statistical analyses were performed using GraphPad Prism software version 9.1.2 (GraphPad Software, CA, USA). One-way ANOVA with Tukey's multiple comparison test was used to compare the means of each group.

\section{Results Induction of cytokine and chemokine production in human primary monocytes by LPS}

To determine the effect of LPS on the activation of human primary monocytes and lymphocytes, we first activated PBMCs with LPS concentrations ranging from 0.01 to $100 \mathrm{ng} / \mathrm{mL}$. The cells were then intracellularly stained for various cytokines (IFN- $\gamma$, TNF- $\alpha$, GM-CSF, IL-1 $\beta$, IL-6, and IL-10) and chemokines (CCL2, CCL3, CCL4, and CXCL10). Cytokine and chemokine production in monocytes and $\mathrm{T}$ lymphocytes was determined by flow cytometry. Gating strategies for monocytes and $\mathrm{T}$ lymphocytes were based on their signature surface molecules and are shown in Fig. 1a.

In monocytes, different cytokines and chemokines exhibited dissimilar responses to LPS stimulation (Fig. 1b, c). In comparison to the unstimulated control, all concentrations of LPS induced the production of proinflammatory cytokines, including TNF- $\alpha, \mathrm{IL}-1 \beta$ and IL-6, but not IL-10, GM-CSF, or IFN- $\gamma$ (Fig. 1d). Among chemokines, the upregulation of CCL3 and CCL4 production was observed in response to $0.1 \mathrm{ng} / \mathrm{mL}$ LPS or higher and $1 \mathrm{ng} / \mathrm{mL}$ LPS or higher, respectively (Fig. 1e). No upregulation of CXCL10 expression was observed at any LPS concentration used (Fig. 1e). Conversely, CCL2 


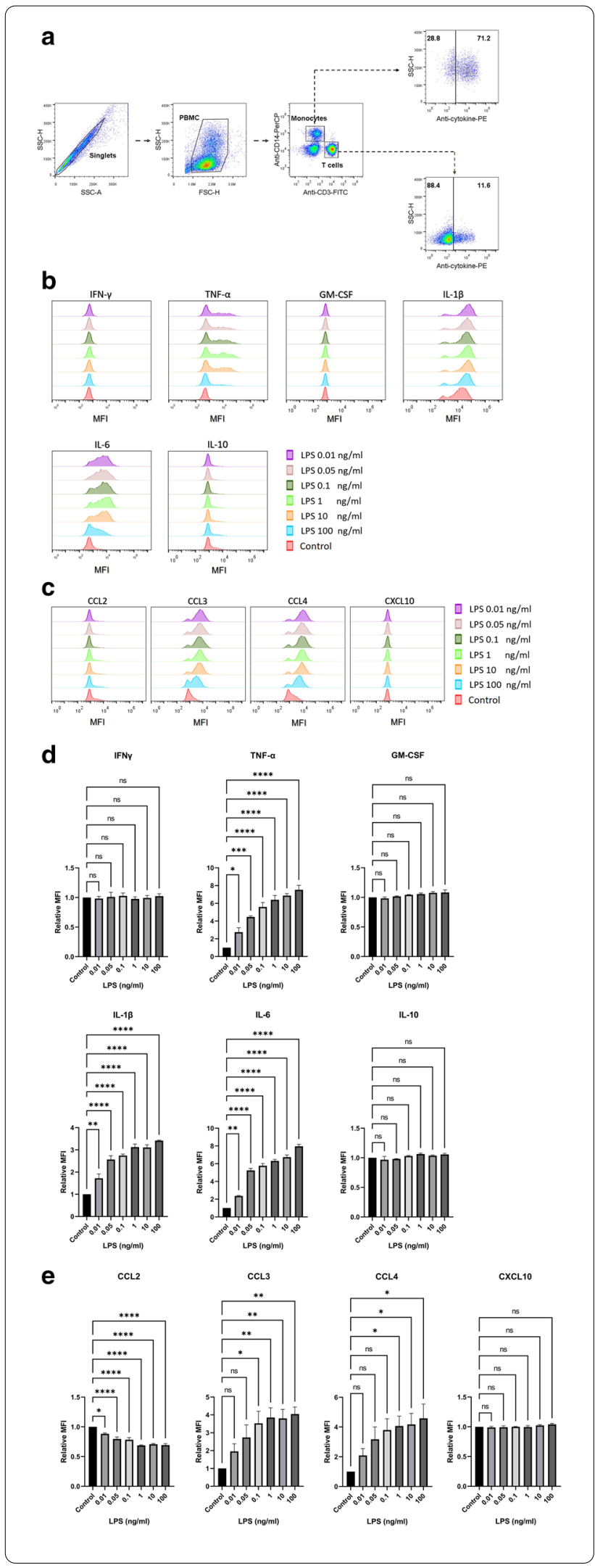

Fig. 1 Lipopolysaccharide induces the production of various cytokines and chemokines in monocytes. PBMCs $(n=3)$ were stimulated with the indicated concentrations of LPS or in the absence of LPS (Control). The intracellular cytokines and chemokines were determined by flow cytometry. The gating strategies on CD14+ monocytes and CD3+T lymphocytes are shown (a). The histogram plots from one of the three individuals on indicated cytokines $(\mathbf{b})$ and chemokines (c) produced in CD14+ monocytes are indicated. The bar graphs indicated relative fluorescent intensity (MFI) of cytokines (d) or chemokines (e) are expressed as mean \pm standard error of mean (SEM). Statistical analysis was performed using one-way ANOVA with Tukey's multiple comparison test. ${ }^{*} ; p \leq 0.05,{ }^{* *} ; p \leq 0.01,{ }^{* * *}$; $p \leq 0.001$, and ${ }^{* * * *} ; p \leq 0.0001$. NS not significant

production was downregulated in response to all concentrations of LPS (Fig. 1e). The flow cytometric profiles of each subject and data analysis are shown in Additional file 1: Fig. S1 and Additional file 5: Table S1).

In $\mathrm{T}$ lymphocytes, no concentrations of LPS affected any tested cytokine or chemokine (Fig. 2a-d). AntiCD3 and anti-CD28 mAbs were used as T lymphocyte activation control, and we found that IFN- $\gamma$ and TNF- $\alpha$ were induced upon $\mathrm{T}$ cell activation (Fig. 2e, f). The flow cytometric profiles of each subject and data analysis are shown in Additional file 2: Fig. S2, Additional file 3: Fig. S3 and Additional file 6: Table S2).

\section{Very low concentrations of LPS induce TNF- $a$ and IL-6 production}

As described above, a low concentration of LPS $(0.01 \mathrm{ng} /$ $\mathrm{mL}$ ) induced the production of TNF- $\alpha$ and IL- 6 . We determined whether even lower concentrations of LPS (0.000625-100 ng/mL) could induce TNF- $\alpha$ and IL-6 production. Surprisingly, at lower concentrations, the effect of LPS on TNF- $\alpha$ and IL- 6 production could still be observed (Fig. 3). Very low concentrations of LPS $(0.0025$ and $0.005 \mathrm{ng} / \mathrm{mL}$ ) could induce TNF- $\alpha$ and IL- 6 production, respectively (Fig. 3a, b). The flow cytometric profiles of each subject and data analysis are shown in Additional file 4: Fig. S4 and Additional file 7: Table S3).

As expected, $\mathrm{T}$ lymphocytes were not activated by LPS, whereas the anti-CD3 and anti-CD28 mAb control exhibited stimulatory effects (data not shown).

\section{Discussion}

LPS is a glycan-based gram-negative pathogen-associated molecular pattern (PAMP) that induces innate immune responses in a sequential manner starting with the binding of LPS to LPS-binding protein, which is then transferred to CD14, and finally interacts with the TLR-4/MD-2 complex [6, 13-15]. Bacterial LPS has 
a
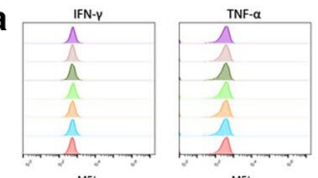

MFi
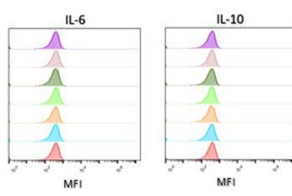

b

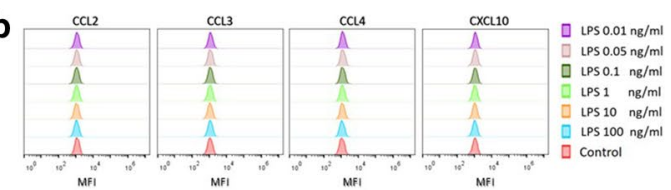

C
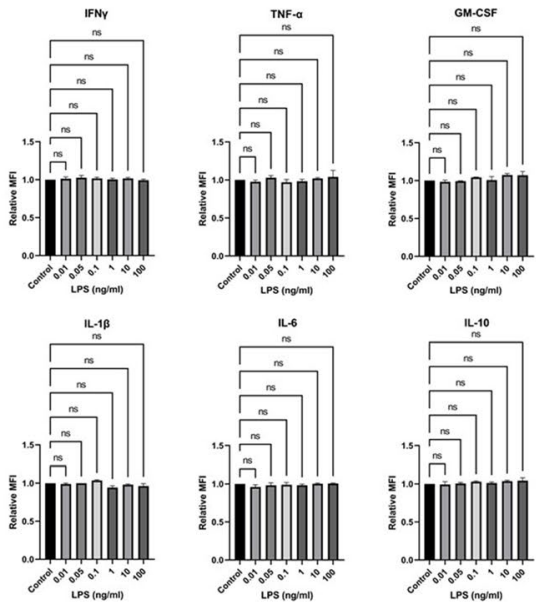

d
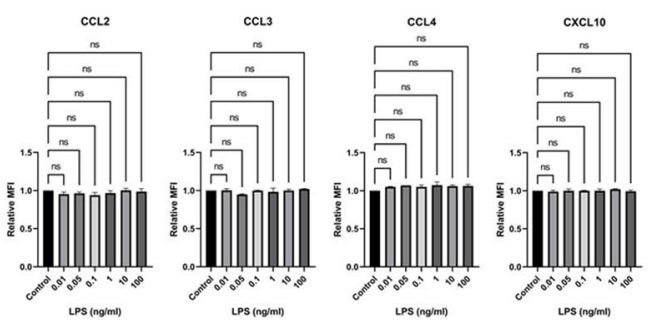

e

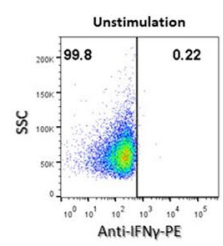

Anti-CD3+ anti-CD28

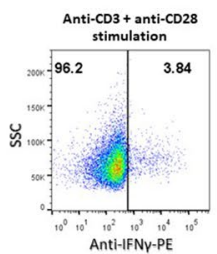

f
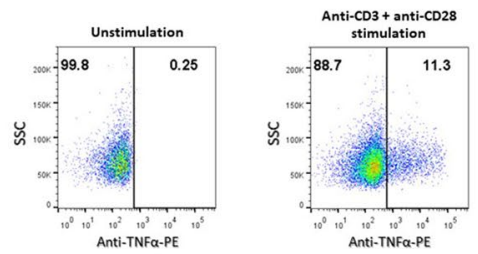

4

Fig. 2 The production of cytokines and chemokines in T lymphocytes upon lipopolysaccharide activation. PBMCs $(n=3)$ were stimulated with the indicated concentrations of LPS or in the absence of LPS (Control). Intracellular cytokines and chemokines were determined using flow cytometry. CD3 + T lymphocyte population was gated and histogram plots from one of the three individuals on indicated cytokines (a) and chemokines (b) are shown. The bar graphs indicated relative mean fluorescent intensity (MFI) of cytokines (c) and chemokines (d) are expressed as mean \pm standard error of mean (SEM). The dot plot showing the expression of IFN- $\gamma(\mathbf{e})$ and TNF-a (f) upon stimulation with anti-CD3 and anti-CD28 mAbs or unstimulated control are shown. Statistical analysis was performed using one-way ANOVA with Tukey's multiple comparison test. NS not significant

been demonstrated to exhibit potent immunostimulatory activity and induce the production of various proinflammatory mediators to control bacterial infection. While several studies have reported the effect of LPS on various cell types $[1,12]$, studies comparing whether various concentrations of LPS affect the production of cytokines and chemokines in human primary monocytes and $\mathrm{T}$ lymphocytes are limited. Because the activation of CD14+ monocytes by LPS leads to the production of cytokines and PBMCs consist of monocytes and lymphocytes, we examined whether while PBMC activation by LPS and the cytokines produced by activated monocytes might have an effect on CD3+ T lymphocytes and induce cytokine production. In the present study, PBMCs were activated with LPS, and cytokine and chemokine production by monocytes and Tlymphocytes was examined.

We demonstrated that LPS could regulate the production of cytokines and chemokines in monocytes. However, LPS did not affect $\mathrm{T}$ lymphocytes. These results indicated that the cytokines and chemokines produced by LPS-activated monocytes have no influence on cytokine production by $\mathrm{T}$ lymphocytes. Surprisingly, we found that a very low concentration of LPS could induce TNF- $\alpha$ and IL- 6 production in monocytes. TNF- $\alpha$ is the prototypical inflammatory cytokine and is involved in several cellular processes, including cytokine and acute phase protein production, adhesion molecule expression, cell proliferation, differentiation, and cell death [1]. IL-6 is also a proinflammatory cytokine. Conversely, IL-6 also plays an anti-inflammatory role $[1,16-18]$. The different activities of IL-6 may depend on the type of signalling that is induced, either through membrane-bound or soluble IL-6R $[1,18]$. TNF- $\alpha$ and IL- 6 thus contribute to many pathophysiological processes, including endotoxic shock $[2,19]$. Schwarz et al. demonstrated that $0.2 \mathrm{ng} / \mathrm{mL}$ LPS could induce the production of TNF- $\alpha$ and IL- 6 by CD14+ 

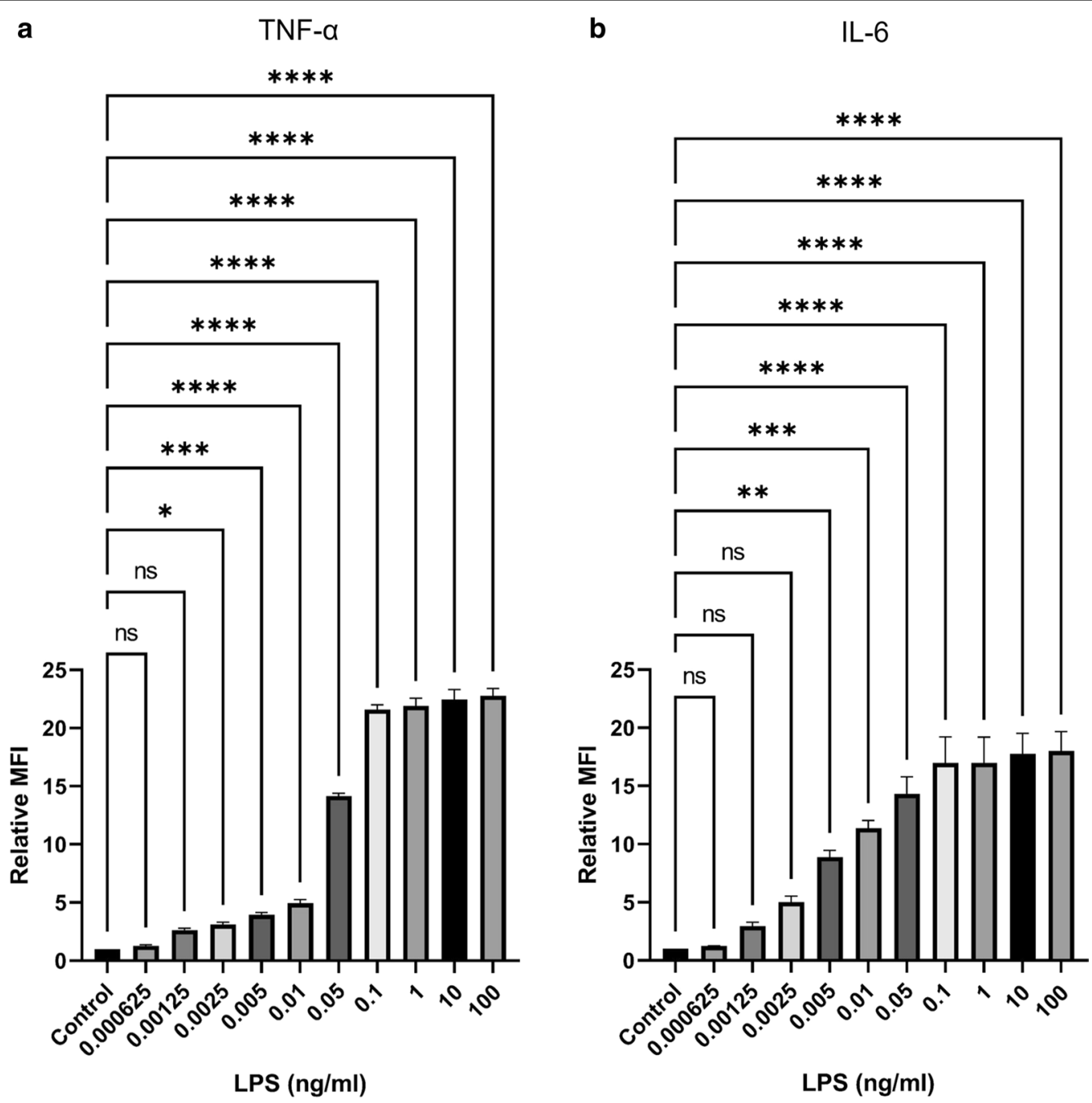

Fig. 3 A very low concentrations of lipopolysaccharide induce the production of TNF-a and IL-6 in monocytes. PBMCs $(n=3)$ were stimulated with the indicated concentrations of LPS or in the absence of LPS (Control). CD14+ monocytes were gated and the bar graphs indicated the relative mean fluorescent intensity (MFI) of TNF- $a(\mathbf{a})$ and IL-6 (b) are expressed as mean \pm standard error of mean (SEM). Statistical analysis was performed using one-way ANOVA with Tukey's multiple

monocytes [12]. Our findings, however, demonstrated that a much lower concentration $(0.0025 \mathrm{ng} / \mathrm{mL})$ of LPS could induce proinflammatory cytokines. The different observations may come from the method used. Purified monocytes were used in the previous study [12], while PBMCs were used in the present study. In addition, secreted cytokines were measured in the previous report [12], while intracellular cytokines were measured in the present study. Similar to the production of proinflammatory cytokines, LPS-induced production of chemokines in innate immune cells has also been reported [1, 20-22]. In agreement with previous reports, our study revealed that LPS is a potent stimulus that induces the production of chemokines. However, the effect of LPS on the induction of chemokines was lower than that observed for cytokines. CCL3 and CCL4 were upregulated in monocytes by 0.1 and $1 \mathrm{ng} /$ mL LPS, respectively. However, LPS had no effect on CXCL10 production and reduced CCL2 production.

LPS stimulates monocytes via innate receptors, including CD14, and the TLR-4/MD-2 complex $[1,4-6,23$, 24]. However, in the absence of CD14, a high concentration of LPS can directly stimulate TLR4 and MD-2 [23, 25]. It was demonstrated that $B$ cells express the receptors RP105 and MD-1, which are structurally related to TLR-4 and MD-2. These receptors are responsible for LPS signalling in B cells $[26,27]$. However, the direct effect of LPS on $\mathrm{T}$ lymphocytes is largely unknown. 
Innate immune stimulation by LPS positively impacts $\mathrm{T}$ lymphocyte priming. LPS stimulation causes antigen presenting cells (APCs) to upregulate MHC class II molecules, costimulatory molecules, and cytokines that boost $\mathrm{T}$ lymphocyte function [23]. A number of publications, controversially, reported evidence for the activation of $T$ lymphocytes by LPS and demonstrate that this activation requires direct cell-to-cell contact with monocytes and costimulatory signals provided by the B7 and CD28 interaction [28-31]. In the present study, however, we found that LPS had no effect on T lymphocytes, which is in line with previous reports [32-34]. The concentrations of LPS and the stimulation time used in our study, however, were different from the reports that showed the effect of LPS on T lymphocytes [28-31].

In summary, we demonstrated that very low concentrations of LPS can induce the production of various cytokines and chemokines in human primary monocytes. This information is important, as low amounts of endotoxin impurities can be found in recombinant proteins produced by $E$. coli [12] and might be sufficient to activate monocytes. These results, therefore, could generate erroneous data when using LPS-contaminated materials to investigate immune functions. To avoid endotoxin contamination, we recommend working with recombinant proteins that have been expressed under endotoxinfree conditions, such as in mammalian cells. In addition, removing LPS from the recombinant proteins using a very effective LPS elimination method is required to avoid the activation of LPS-sensitive immune cells.

\section{Limitations}

We demonstrated that LPS have no effect on T lymphocytes. However, in our study, PBMCs were incubated with LPS for only $6 \mathrm{~h}$. Additional investigations using longer incubation times of LPS and PBMCs are required to confirm that LPS has no effect on $\mathrm{T}$ lymphocytes among PBMCs.

\footnotetext{
Abbreviations

BSA: Bovine serum albumin; CCL: Chemokine (C-C motif) ligand; CD: Cluster of differentiation; CXCL: Chemokine (C-X-C motif) ligand; FBS: Fetal bovine serum; FITC: Fluorescein isothiocyanate; FSC: Forward scatter; GM-CSF: Granulocyte-macrophage colony-stimulating factor; IFN: Interferon; IL: Interleukin; IL-6R: Interleukin-6 receptor; IRF3: Interferon regulatory transcription factor 3; MD-2: Myeloid differentiation 2; MHC: Major histocompatibility complex; MyD88: Myeloid differentiation primary response 88; NF-KB: Nuclear factor kappa light chain enhancer of activated B cells; PE: Phycoerythrin; PerCP: Peridinin Chlorophyll Protein Complex; RP105: Radioprotective 105 kDa; SSC: Side scatter; TLR: Toll-like receptor; TNF: Tumor necrosis factor; TRIF: TIR (Toll/interleukin-1 receptor) domain-containing adaptor protein inducing interferon beta.
}

\section{Supplementary Information}

The online version contains supplementary material available at https://doi. org/10.1186/s13104-022-05941-4.

Additional file 1: Figure S1. Flow cytometric profiles of each subject (according to Fig. 1 in the paper): lipopolysaccharide induces the production of various cytokines and chemokines in monocytes. PBMCs were stimulated with the indicated concentrations of LPS or in the absence of LPS (Control). The intracellular cytokines and chemokines were determined by flow cytometry. CD14+ monocyte population of the three individuals (as indicated) were gated and dot plotted on the expression of the indicated cytokines and chemokines are shown (numbers indicate the $\%$ cells).

Additional file 2: Figure S2. Flow cytometric profiles of each subject (according to Fig. 2 in the paper): the production of cytokines and chemokines in Tlymphocytes upon lipopolysaccharide activation. PBMCs $(n=3)$ were stimulated with the indicated concentrations of LPS or in the absence of LPS (Control). Intracellular cytokines and chemokines were determined using flow cytometry. CD3+T lymphocyte population of the three individuals (as indicated) were gated and dot plotted on the expression of the indicated cytokines and chemokines are shown (numbers indicate the $\%$ cells).

Additional file 3: Figure S3. Flow cytometric profiles of each subject (according to Fig. 2 in the paper): the production of TNF- $\alpha$ and IFN- $\gamma$ in T lymphocytes upon anti-CD3 and anti-CD28 mAbs activation. PBMCs were stimulated with anti-CD3 and anti-CD28 mAbs or or unstimulated control (as indicated). Intracellular cytokines were determined using flow cytometry. CD3 + T lymphocyte population of the three individuals (as indicated) were gated and dot plotted on the expression of the TNF-a and IFN- $\gamma$ upon stimulation with anti-CD3 and anti-CD28 mAbs or unstimulated control are shown (numbers indicate the $\%$ cells).

Additional file 4: Figure S4. Flow cytometric profiles of each subject (according to Fig. 3 in the paper): lipopolysaccharide induces the production of TNF- $a$ and IL- 6 in monocytes. PBMCs were stimulated with the indicated concentrations of LPS or in the absence of LPS (Control). The intracellular TNF- $a$ and IL-6 were determined by flow cytometry. CD14+ monocyte population of the three individuals (as indicated) were gated and dot plotted on the expression of the indicated cytokines are shown (numbers indicate the \% cells).

Additional file 5: Table S1. Data analyzed form flow cytometric profiles of each subject (according to Fig. 1 in the paper): lipopolysaccharide induces the production of various cytokines and chemokines in monocytes. PBMCs were stimulated with the indicated concentrations of LPS. The intracellular cytokines and chemokines were determined by flow cytometry. CD14+ monocyte population of the three individuals (as indicated) were gated and mean fluorescence intensity of the expression of the indicated cytokines and chemokines are shown.

Additional file 6: Table S2. Data analyzed form flow cytometric profiles of each subject (according to Fig. 2 in the paper): lipopolysaccharide induces the production of various cytokines and chemokines in T lymphocytes. PBMCs were stimulated with the indicated concentrations of LPS. The intracellular cytokines and chemokines were determined by flow cytometry. CD3+T lymphocyte population of the three individuals (as indicated) were gated and mean fluorescence intensity of the expression of the indicated cytokines and chemokines are shown.

Additional file 7: Table S3. Data analyzed form flow cytometric profiles of each subject (according to Fig. 3 in the paper): lipopolysaccharide induces the production of TNF- $a$ and IL- 6 in monocytes. PBMCs were stimulated with the indicated concentrations of LPS. The intracellular TNF- $a$ and IL- 6 were determined by flow cytometry. CD14+ monocyte population of the three individuals (as indicated) were gated and mean fluorescence intensity of the expression of the indicated cytokines are shown. 


\section{Acknowledgements}

We are thankful to Miss Kantinan Chuensirikulchai and Miss Sasiwimon Chaikum for their technical assistance.

\section{Authors' contributions}

RC performed experiments, data analysis and drafted the original manuscript. WK, the principal investigator, provided a conceptual framework for the project, provided guidance for methodology and interpretation of the data, edited and finalized the manuscript. Both authors read and approved the final manuscript.

\section{Funding}

This work was supported by the Distinguished Research Professor Grant (NRCT808/2563) of the National Research Council of Thailand. This research project was also partially supported by TSRI and Chiang Mai University Center of Excellence Project. RC obtained scholarship supported by Department of Medical Technology, Faculty of Associated Medical Sciences, Chiang Mai University.

\section{Availability of data and materials}

All data generated or analyzed during the current study are available in Additional files.

\section{Declarations}

\section{Ethics approval and consent to participate}

This study was approved by the Ethics Committee of the Faculty of Associated Medical Sciences, Chiang Mai University, Thailand (AMSEC-64EX-016). Human peripheral blood was obtained from healthy Thai adult volunteers after receiving written informed consent. All experiments were performed in accordance with the relevant guidelines and regulations.

\section{Consent for publication}

Not applicable.

\section{Competing interests}

The authors declare that they have no competing interests.

\section{Author details}

${ }^{1}$ Division of Clinical Immunology, Department of Medical Technology, Faculty of Associated Medical Sciences, Chiang Mai University, Chiang Mai, Thailand. ${ }^{2}$ Biomedical Technology Research Center, National Center for Genetic Engineering and Biotechnology, National Science and Technology Development Agency at the Faculty of Associated Medical Sciences, Chiang Mai University, Chiang Mai, Thailand.

Received: 21 November 2021 Accepted: 31 January 2022

Published online: 10 February 2022

\section{References}

1. Rossol M, Heine H, Meusch U, Quandt D, Klein C, Sweet MJ, et al. LPSinduced cytokine production in human monocytes and macrophages. Crit Rev Immunol. 2011;31(5):379-446.

2. Morris MC, Gilliam EA, Li L. Innate immune programing by endotoxin and its pathological consequences. Front Immunol. 2014;5:680.

3. Rathinam VAK, Zhao Y, Shao F. Innate immunity to intracellular LPS. Nat Immunol. 2019;20(5):527-33.

4. Takeda K, Akira S. TLR signaling pathways. Semin Immunol. 2004;16(1):3-9.

5. Zughaier SM, Zimmer SM, Datta A, Carlson RW, Stephens DS. Differential induction of the toll-like receptor 4-MyD88-dependent and -independent signaling pathways by endotoxins. Infect Immun. 2005;73(5):2940-50.

6. Ryu JK, Kim SJ, Rah SH, Kang Jl, Jung HE, Lee D, et al. Reconstruction of LPS transfer cascade reveals structural determinants within LBP, CD14, and TLR4MD2 for efficient LPS recognition and transfer. Immunity. 2017;46(1):38-50.
7. Bertani B, Ruiz N. Function and biogenesis of lipopolysaccharides. EcoSal Plus. 2018. https://doi.org/10.1128/ecosalplus.ESP-0001-2018.

8. Zamyatina A, Heine $\mathrm{H}$. Lipopolysaccharide recognition in the crossroads of TLR4 and caspase-4/11 mediated inflammatory pathways. Front Immunol. 2020;11: 585146.

9. Alexander C, Rietschel ET. Bacterial lipopolysaccharides and innate immunity. J Endotoxin Res. 2001;7(3):167-202.

10. Conti P, Dempsey RA, Reale M, Barbacane RC, Panara MR, Bongrazio M, et al. Activation of human natural killer cells by lipopolysaccharide and generation of interleukin-1 alpha, beta, tumour necrosis factor and interleukin-6. Effect of IL-1 receptor antagonist. Immunology. 1991;73(4):450-6.

11. Dawson M, Novitsky T, Gould M. Microbes, endotoxins and water. Pharm Eng. 1988;8(2):145-8.

12. Schwarz H, Schmittner M, Duschl A, Horejs-Hoeck J. Residual endotoxin contaminations in recombinant proteins are sufficient to activate human CD1c+ dendritic cells. PLoS ONE. 2014;9(12): e113840.

13. Zubova SV, Grachev SV, Prokhorenko IR. Effect of lipopolysaccharide structure on functional response of whole blood cells. Immunobiology. 2021;226(1): 152030 .

14. Landmann R, Knopf HP, Link S, Sansano S, Schumann R, Zimmerli W. Human monocyte CD14 is upregulated by lipopolysaccharide. Infect Immun. 1996;64(5):1762-9.

15. Roslansky PF, Novitsky TJ. Sensitivity of limulus amebocyte lysate (LAL) to LAL-reactive glucans. J Clin Microbiol. 1991;29(11):2477-83.

16. Xing Z, Gauldie J, Cox G, Baumann H, Jordana M, Lei XF, et al. IL-6 is an antiinflammatory cytokine required for controlling local or systemic acute inflammatory responses. J Clin Invest. 1998;101(2):311-20.

17. Ulich TR, Yin S, Guo K, Yi ES, Remick D, del Castillo J. Intratracheal injection of endotoxin and cytokines. II. Interleukin-6 and transforming growth factor beta inhibit acute inflammation. Am J Pathol. 1991;138(5):1097-101.

18. Scheller J, Chalaris A, Schmidt-Arras D, Rose-John S. The pro- and antiinflammatory properties of the cytokine interleukin-6. Biochim Biophys Acta. 2011;1813(5):878-88.

19. Morrison DC, Ryan JL. Endotoxins and disease mechanisms. Annu Rev Med. 1987;38:417-32

20. Hirohashi N, Morrison DC. Low-dose lipopolysaccharide (LPS) pretreatment of mouse macrophages modulates LPS-dependent interleukin-6 production in vitro. Infect Immun. 1996:64(3):1011-5.

21. Chaudhry H, Zhou J, Zhong Y, Ali MM, McGuire F, Nagarkatti PS, et al. Role of cytokines as a double-edged sword in sepsis. In vivo (Athens, Greece) 2013;27(6):669-84.

22. Leon LR, White AA, Kluger MJ. Role of IL-6 and TNF in thermoregulation and survival during sepsis in mice. Am J Physiol. 1998;275(1):R269-77.

23. McAleer JP, Vella AT. Understanding how lipopolysaccharide impacts CD4 T-cell immunity. Crit Rev Immunol. 2008;28(4):281-99.

24. Pålsson-McDermott EM, O'Neill LA. Signal transduction by the lipopolysaccharide receptor, Toll-like receptor-4. Immunology. 2004;113(2):153-62.

25. Haziot A, Ferrero E, Köntgen F, Hijiya N, Yamamoto S, Silver J, et al. Resistance to endotoxin shock and reduced dissemination of gram-negative bacteria in CD14-deficient mice. Immunity. 1996;4(4):407-14

26. Ogata H, Su I, Miyake K, Nagai Y, Akashi S, Mecklenbräuker I, et al. The toll-like receptor protein RP105 regulates lipopolysaccharide signaling in B cells. J Exp Med. 2000;192(1):23-9.

27. Nagai Y, Shimazu R, Ogata H, Akashi S, Sudo K, Yamasaki H, et al. Requirement for MD-1 in cell surface expression of RP105/CD180 and B-cell responsiveness to lipopolysaccharide. Blood. 2002;99(5):1699-705.

28. Ulmer AJ, Flad H, Rietschel T, Mattern T. Induction of proliferation and cytokine production in human T lymphocytes by lipopolysaccharide (LPS). Toxicology. 2000;152(1-3):37-45.

29. Mattern T, Thanhäuser A, Reiling N, Toellner KM, Duchrow M, Kusumoto S, et al. Endotoxin and lipid A stimulate proliferation of human T cells in the presence of autologous monocytes. J Immunol. 1994;153(7):2996-3004.

30. Mattern T, Flad HD, Brade L, Rietschel ET, Ulmer AJ. Stimulation of human T lymphocytes by LPS is MHC unrestricted, but strongly dependent on B7 interactions. J Immunol. 1998;160(7):3412-8.

31. Mattern T, Girroleit G, Flad HD, Rietschel ET, Ulmer AJ. CD34(+) hematopoietic stem cells exert accessory function in lipopolysaccharide-induced 
T cell stimulation and CD80 expression on monocytes. J Exp Med. 1999;189(4):693-700.

32. Greaves M, Janossy G, Doenhoff M. Selective triggering of human T and B lymphocytes in vitro by polyclonal mitogens. J Exp Med. 1974;140(1):1-18.

33. Miller RA, Gartner S, Kaplan HS. Stimulation of mitogenic responses in human peripheral blood lymphocytes by lipopolysaccharide: serum and T helper cell requirements. J Immunol. 1978;121(6):2160-4.

34. Schmidtke JR, Najarian JS. Synergistic effects on DNA synthesis of phytohemagglutinin or concanavalin A and lipopolysaccharide in human peripheral blood lymphocytes. J Immunol. 1975;114(2 pt 2):742-6.

\section{Publisher's Note}

Springer Nature remains neutral with regard to jurisdictional claims in published maps and institutional affiliations.

- fast, convenient online submission

- thorough peer review by experienced researchers in your field

- rapid publication on acceptance

- support for research data, including large and complex data types

- gold Open Access which fosters wider collaboration and increased citations

- maximum visibility for your research: over $100 \mathrm{M}$ website views per year

At BMC, research is always in progress.

Learn more biomedcentral.com/submissions 